\title{
First day-time seeing observations at the TÜBITAK National Observatory in Turkey
}

\author{
T. Özış1k ${ }^{1}$ and T. Ak ${ }^{2}$ \\ 1 TÜBİTAK National Observatory, Akdeniz University Campus, 07058 Antalya, Turkey \\ e-mail: tuncay@tug.tug.tubitak.gov.tr \\ 2 Istanbul University Observatory Research and Application Center, 34119 Istanbul, Turkey \\ e-mail: tanselak@istanbul.edu.tr
}

Received 25 February 2004 / Accepted 30 April 2004

\begin{abstract}
Despite the fact that selecting good solar observatory sites is an important matter, day-time seeing quality of observatory sites in Turkey has not been previously recorded. Therefore, we built a Solar Differential Image Motion Monitor (S-DIMM) for day-time seeing observations at the TÜBİTAK National Observatory (TUG) in Turkey which is being tested as a likely site for a future large aperture solar telescope. In this study, we describe the design of TUG S-DIMM instrument and a reduction procedure for its data. First results obtained at $7 \mathrm{~m}$ above ground show that the day-time Fried parameters at the TUG site are comparable with those of some solar observatory sites. The best seeing conditions at the TUG site occur between October and December with a median Fried parameter of $6.95 \mathrm{~cm}$ in the first three hours of the day. The median Fried parameter calculated from all observations made during our observation period is $4.48 \mathrm{~cm}$. Our observations also reveal that the best day-time Fried parameters are obtained for wind speeds higher than $3 \mathrm{~m} / \mathrm{s}$.
\end{abstract}

Key words. atmospheric effects - turbulence - site testing

\section{Introduction}

Earth's atmosphere causes random motion of the diffractionlimited image formed by a telescope. Random fluctuations in the index of refraction throughout the Earth's atmosphere result in image degradation. This effect is generally called as "seeing" and limits the telescope's angular resolution. Fried parameter $r_{0}$ (Fried 1965) describes all seeing effects and it can be measured from the image motion in a small telescope (Tokovinin 2002).

Direct measurements of image motion in the focal plane of a telescope are perturbed with the motion induced by telescope vibrations due to tracking errors and wind shakes (Martin 1987). In contrast, differential image motion measurements do not suffer from the telescope vibrations. Differential image motion produced by turbulence can be measured with a DIMM (Differential Image Motion Monitor) (Sarazin \& Roddier 1990; Tokovinin 2002).

Although the DIMM concept was first introduced by Stock \& Keller (1960), differential image motion monitors have been built by many observatories for image motion measurements since the modern implementation of the DIMM concept was described by Sarazin \& Roddier (1990) and Vernin \& Muñoz-Tuñon (1995). In DIMM principle, light that passes through two separated small apertures of a Hartmann mask is separated by means of an optical wedge in one of the apertures before falling on a detector. This produces two well separated images of the same star on the detector. Thus, telescope vibrations affect each sub-image in the same way while turbulence induces differential motions in the sub-images. Variations in the image separations can be used to obtain a quantitative estimate of the Fried parameter (Bally et al. 1996).

The Solar - Differential Image Motion Monitor (S-DIMM) is based on the DIMM and it is used for day-time seeing studies by attaching a solar filter to the DIMM. Differential motions of the solar limb are observed with the S-DIMM in order to calculate the day-time Fried parameter (Beckers 2001).

Since day-time seeing conditions of observatory sites in Turkey have not been known, we built a S-DIMM for daytime differential image motion observations at the TÜBİTAK National Observatory (hereafter referred to as TUG) in Turkey. Only a few S-DIMMs have been developed until the TUG S-DIMM described in this study was constructed. The first one was built at the Yunnan Observatory (Liu \& Beckers 2000) and others were built for site testing studies of ATST (Advanced Technology Solar Telescope) (Beckers 1998; Keil et al. 2002).

In this paper, we describe the design of TUG S-DIMM instrument and a reduction procedure for TUG S-DIMM data. We also give the first results of the observations and compare them with those obtained at other observatory sites. 
Table 1. The components and instrumental parameters of the TUG S-DIMM.

\begin{tabular}{lc}
\hline \hline Telescope & Meade LX200 SCT \\
Model & $304.8 \mathrm{~mm}$ \\
Aperture & $3048 \mathrm{~mm}$ \\
Focal length & \\
\hline Hartmann mask & $40 \mathrm{~mm}$ \\
Aperture diameter $(D)$ & $250 \mathrm{~mm}$ \\
Aperture separation $(d)$ & $60 \mathrm{~mm}$ \\
\hline Optical wedge & $80^{\prime \prime}$ \\
Diameter & \\
Deviation angle & $10^{-5}$ \\
\hline Solar filter & \\
Model & $2^{+}$, Yellow-Orange \\
Type & Thousand Oaks Glass Solar Filter \\
Transparency & $0.001 \mathrm{~s}$ \\
\hline Video CCD camera & $25 \mathrm{~Hz}$ \\
Model & \\
Exposure time & Astrovid StellaCam EX (PAL) \\
Cadence & \\
\hline Digital video recorder & SONY GV-D800E Digital Recorder \\
Model & Digital8 video cassette \\
Recording media &
\end{tabular}

\section{The instrument}

\subsection{Instrumental parameters and location}

TUG S-DIMM is based on a Meade LX200 SCT telescope with a $304.8 \mathrm{~mm}$ clear aperture and a f/10 focal rate. The aperture is covered by a neutral density solar filter with $10^{-5}$ transmittance in visible and a Hartmann mask with two circular holes of $40 \mathrm{~mm}$ diameter. The separation of the holes is $250 \mathrm{~mm}$. A wedge prism with a deviation angle of $80^{\prime \prime}$ is located in one of the Hartmann holes. The detector at the focal plane is an Astrovid StellaCam EX astronomical video CCD camera which has composite video output. The video signal is recorded with a digital video recorder at a speed of $25 \mathrm{fps}$. Using this type of data record, we gain a huge data capacity during the observations, as done in Bally et al. (1996). The data is then transferred to a computer's disk by means of a professional video capture card for data reduction in DVD (Digital Versatile Disk) format. The components and instrumental parameters of the TUG S-DIMM are summarized in Table 1.

The wedge prism produces two well separated images of the solar limb on the detector as done in the stellar DIMMs. The variable position differences of the two limbs are used to measure the solar differential image motions. Unlike the stellar images, solar differential image motions can only be measured in one direction. We used the longitudinal (i.e., parallel to apertures vector of the Hartmann mask) direction for the image motion. Thus, the apertures vector was located so that its direction is vertical to the horizon.

The TUG S-DIMM was located on the south-east corner of the terrace of T40 photometric telescope building. With the height of the Meade telescope field tripod and the telescope itself, the resulting effective height of the TUG S-DIMM from the ground is $7 \mathrm{~m}$.

\subsection{The plate scale, $d / D$ rate, exposure time and the Strehl ratio}

In order to calibrate the S-DIMM measurements from limb displacements to $r_{0}$ in $\mathrm{cm}$, the image scale of the S-DIMM must be known. Removing the solar filter and wedge prism, the plate scale was found by repeadetly observing stellar pairs with known separations through the TUG S-DIMM optical system. We determined an image scale of $0.56 \pm 0.01^{\prime \prime} /$ pixel from the analysis of this data. This value matches the theoretical calculation.

Differential image motion monitors are not sensitive to wavefront perturbations that are smaller than the aperture or larger than the separation of the pupils on the Hartmann mask (Tokovinin 2002). According to Sarazin \& Roddier (1990), $b=d / D$ rate should be larger than 2.5 ; here $D$ is the aperture diameter and $d$ aperture separation. For the instrument described here, this rate is 6.25 which is a very good value.

In addition to $b=d / D$ rate, exposure time of the detector is another crucial parameter for seeing studies. It should be shorter than $0.01 \mathrm{~s}$ to freeze the atmospheric image motion (Martin 1987). Thus, the exposure time of the video ccd camera is adjusted to a constant value of $0.001 \mathrm{~s}$.

Strehl ratio of the both stellar spots produced by a differential image motion monitor can be used to monitor the optical quality of the instrument. If $I_{\max }$ is the maximum pixel intensity in a spot and $I_{\text {tot }}$ is the total flux, the Strehl ratio can be estimated as (Tokovinin 2002)

$S=\frac{I_{\max }}{I_{\text {tot }}} \frac{4}{\pi}\left(\frac{\lambda_{\mathrm{ccd}}}{D \Delta x}\right)^{2}$

where $\Delta x$ is the CCD pixel size in radians and $\lambda_{\text {ccd }}$ the maximum CCD sensitivity. Removing the solar filter, we observed a bright star while the Fried parameter was $\sim 10 \mathrm{~cm}$ and estimated a Strehl ratio of $0.68 \pm 0.02$. Since the typical empirical values of the Strehl ratio are between 0.4 and 0.7 , stellar observations reveal that the TUG S-DIMM has quite good optics.

\section{Observations and data reduction}

TÜBİTAK National Observatory (latitude $36^{\circ} 49^{\prime} \mathrm{N}$, longitude $30^{\circ} 20^{\prime} \mathrm{E}$ ) is located at the south of Turkey at a distance of about $50 \mathrm{~km}$ south-west of Antalya. It lies on a branch of the Taurus Mountain System at $2550 \mathrm{~m}$ above sea level. The observatory has three telescopes with apertures of $1.5 \mathrm{~m}, 0.45 \mathrm{~m}$ and $0.4 \mathrm{~m}$ for stellar observations. This site is being tested as a likely site for a future large aperture solar telescope.

The observations were done in the days of April 16, May 9, 22, 23, June 23, 24, 25, 26, July 21, 22, August 23, 24, 25, 26, September 19, 20, 21, 22, 23, October 15, 16, November 17 and December 28, 29, 2003 at the TUG site. In clear days, the time interval of observations was 04:00-14:00 UT. Note that May and July observations cover only 04:00-09:00 UT due to weather conditions. The video sequences were recorded with 15 min intervals during the observations. The duration of each record is about $20 \mathrm{~s}$ allowing 500 frames for a single Fried parameter estimation. We also recorded the average ground level wind speed, wind direction, temperature, and humidity using 


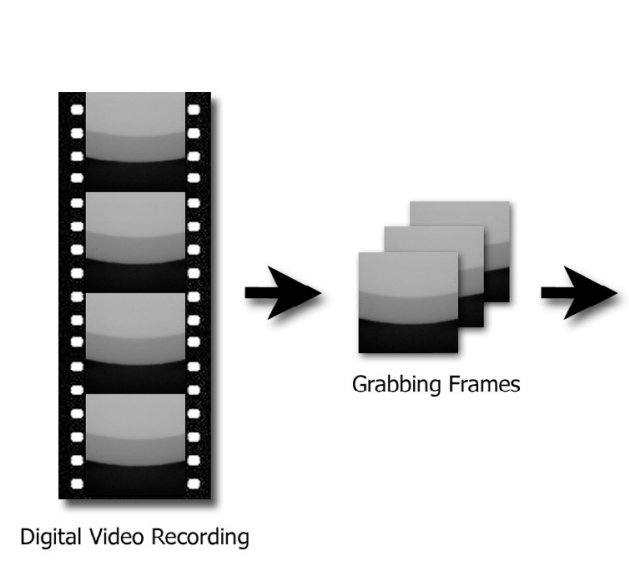

Digital Video Recording

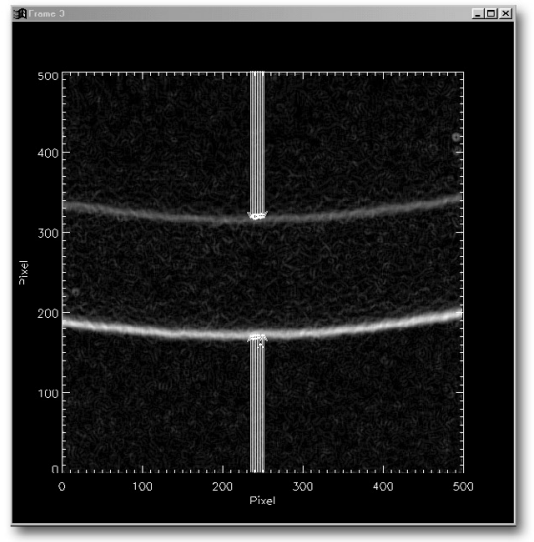

Flat Fielding + Sobel Filtering + Digital Slit

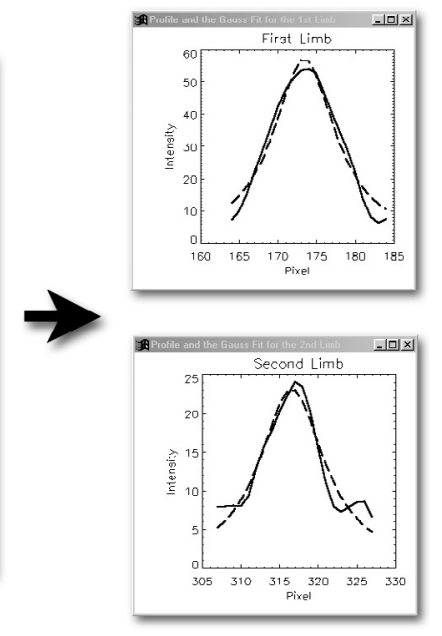

Limb Profiles and Gaussian Fits

Fig. 1. Basic processing steps of the TUG S-DIMM data reduction software.

meteorological instruments placed where the TUG S-DIMM is located.

We developed a complete data reduction algorithm in IDL (Interactive Data Language) for which basic processing procedure is illustrated in Fig. 1. The reduction steps of the TUG S-DIMM data are as follows:

1. Grabbing limb and flat field frames in FITS format from digital video records.

2. Creating a superflat image and correcting frames for the flat field.

3. Applying Median Smooth and Sobel Filters to each frame for the reduction of noise and edge enhancement, respectively.

4. Selecting a limb profile direction on the first frame. This position is constant for all frames grabbed from the video record.

5. Finding the limb positions along the profile line by means of Gaussian fits.

6. Repeating the last step for 8 different profile directions one pixel apart from each other: 4 directions to the right and 4 directions to the left of the first selected direction. By this way, we create a digital slit of $10^{\prime \prime}$ in width.

7. Averaging the limb positions found in the last two steps for each limb.

8. Finding the difference between the average positions of the first and second limb. This difference is the separation of the solar limbs in the first frame.

9. Repeating steps 5-8 for the remaining 499 frames.

10. Calculating the variance $\left(\sigma^{2}\right)$ of the limb separations.

Variance of the separations is the variance of the solar differential image motion that can be used to calculate the Fried parameter with the following formula (Tokovinin 2002):

$\sigma^{2}=K_{l} \lambda^{2} r_{0}^{-5 / 3} D^{-1 / 3}$.

Here $K_{l}$ is a constant (longitudinal Zernike tilt), $\lambda$ is the wavelength, $r_{0}$ is the Fried parameter and $D$ is the diameter of the Hartmann holes. The constant $K_{l}$ is defined as

$K_{l}=0.364\left(1-0.532 b^{-1 / 3}-0.024 b^{-7 / 3}\right)$ where $b=d / D$. Once we calculate the Fried parameter, seeing can be estimated with the following standard formula:

$\epsilon_{0}=0.98 \frac{\lambda}{r_{0}}$

All of the steps listed above give a single seeing, or the Fried parameter, estimation. Since we use only longitudinal image motion measurements, formulae for transverse differential image motion measurements are not given here.

\section{First results and comparison}

\subsection{Meteorological conditions}

The number of clear days is an important parameter in selecting solar observatory sites. Unfortunately, there are no meteorological statistics showing the number of clear days at TUG. Therefore, we monitored the clear days at the observatory for the period of June-December, 2003. Here, the term "clear day" shows the days for which the cloudiness is not more than $5 \%$ from sunrise to sunset. Our observations reveal that $35 \%$ of this period is clear and that the longest string of clear days is 7 . Percentage of continuously clear time of $4 \mathrm{~h}$ before noon is around $50 \%$. Clearly, it rises much higher, around $80 \%$, for periods of continuous sunshine of $1 \mathrm{~h}$ or more. The longest string of continuously clear time of $4 \mathrm{~h}$ before noon is 16 . The longest string of clear days were found 10 for Tucson (USA), 21 for Mt. Wilson (USA), 11 for Big Bear (USA), 13 for Mauna Loa (Hawaii), 24 for Learmonth (Australia), 2 for Urumqi (China), 13 for Udaipur (India) and 17 for El Teide (Spain) (Ambastha et al. 1991; Hill et al. 1994). Although we do not have even one year long record for meteorological observations, these values imply that further meteorological observations can reveal good day-time conditions at the TUG site from the point of view of number of clear days. It should be noted that the meteorological conditions at the TUG site in winter season of the year 2003 were very hard compared to last decades.

Meteorological records taken during observations show that dominant winds at the TUG blow from the East (28\%) 
Table 2. The minimum, maximum and mean values for ground level wind speed and temperature during the observation period.

\begin{tabular}{lcccccc}
\hline \hline Month & \multicolumn{3}{c}{$\begin{array}{c}\text { Wind speed } \\
(\mathrm{m} / \mathrm{s})\end{array}$} & \multicolumn{3}{c}{$\begin{array}{c}\text { Temperature } \\
\left({ }^{\circ} \mathrm{C}\right)\end{array}$} \\
\hline & Min. & Max. & Mean & Min. & Max. & Mean \\
\hline Apr. & 0 & 3.1 & 1.1 & 4.4 & 10.9 & 6.9 \\
May & 0 & 2.0 & 1.0 & 6.5 & 16.4 & 10.1 \\
Jun. & 0.8 & 4.3 & 1.9 & 10.6 & 23.4 & 17.5 \\
Jul. & 0.9 & 2.7 & 1.8 & 11.9 & 20.5 & 15.7 \\
Aug. & 0.6 & 2.8 & 1.4 & 12.5 & 23.7 & 17.5 \\
Sep. & 0 & 3.9 & 1.5 & 5.1 & 20.1 & 13.7 \\
Oct. & 0.7 & 2.9 & 1.7 & 8.6 & 16.7 & 12.7 \\
Nov. & 0.9 & 4.4 & 2.5 & 0.6 & 8.4 & 5.5 \\
Dec. & 0 & 1.5 & 0.6 & -6.2 & 10.7 & 3.4 \\
\hline & & & & & &
\end{tabular}

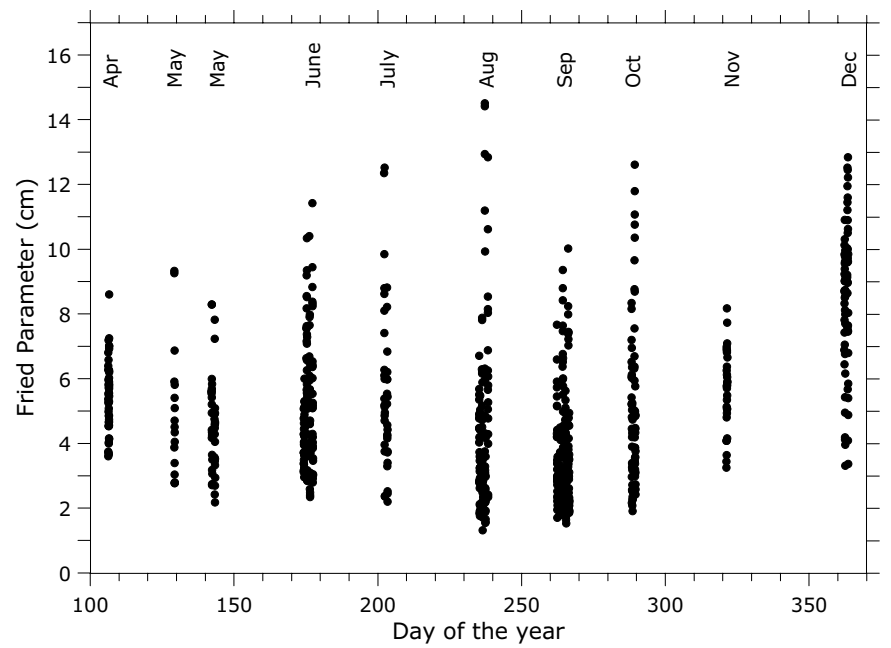

Fig. 2. Fried parameters obtained with the TUG S-DIMM during the observation period. Error bars are omitted for clarity.

and North-East (22\%). Through the S-DIMM observations, we also measured 15 min means of wind speed, temperature and humidity between 04:00-14:00 UT. We list the minimum, maximum and mean values of wind speed and temperature in Table 2 for the observation period. As can be seen from Table 2, wind speeds during the observations were remarkably low for a mountain site. Humidity measurements at the site demonstrate that the TUG site has moderate $(30-50 \%)$ humidity for the observation period.

\subsection{The Fried parameter}

Fried parameters obtained with the TUG S-DIMM during the period of April-December are shown in Fig. 2. May 9, 22, 23 and July 21, 22 were clouded out after 09:00 UT. Thus, the Fried parameters measured on those days cover only 04:00-09:00 UT time interval.

Cumulative frequency distribution of the Fried parameters is demonstrated in Fig. 3. We find that about $5 \%$ of Fried parameters obtained from observations is larger than $10 \mathrm{~cm}$. The monthly median Fried parameters with their first and third quartiles are given in Table 3. As can be seen from Table 3, the best seeing conditions were found between October and

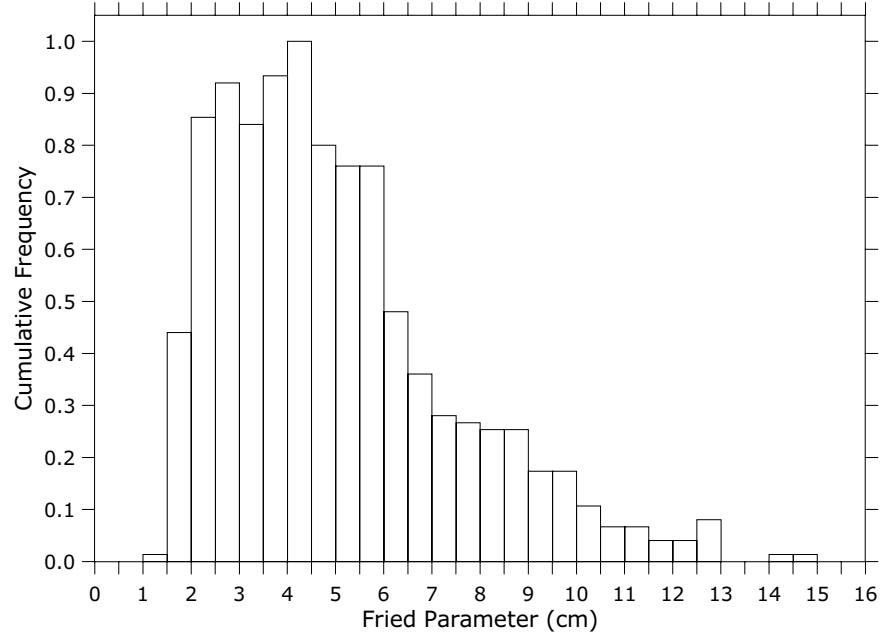

Fig. 3. Cumulative frequency distribution of the day-time Fried parameter at the TUG site for the observation period.

Table 3. Monthly median Fried parameters $\left(r_{0}\right)$ for the observation period. First $\left(q_{1}\right)$ and third $\left(q_{3}\right)$ quartiles are also given. Note that May and July observations cover only 04:00-09:00 UT.

\begin{tabular}{lccccc}
\hline \hline Month & Day & Hours & $\begin{array}{c}q_{1} \\
(\mathrm{~cm})\end{array}$ & $\begin{array}{c}r_{0} \\
(\mathrm{~cm})\end{array}$ & $\begin{array}{c}q_{3} \\
(\mathrm{~cm})\end{array}$ \\
\hline Apr. & 1 & 9 & 4.65 & 5.55 & 6.19 \\
May & 3 & 15 & 3.47 & 4.44 & 5.48 \\
Jun. & 4 & 30 & 3.71 & 4.73 & 6.30 \\
Jul. & 2 & 10 & 3.96 & 5.17 & 6.83 \\
Aug. & 4 & 30 & 2.34 & 3.24 & 5.05 \\
Sep. & 5 & 44 & 2.41 & 3.41 & 4.45 \\
Oct. & 2 & 19 & 3.22 & 4.44 & 6.09 \\
Nov. & 1 & 7 & 4.93 & 5.77 & 6.65 \\
Dec. & 2 & 17 & 7.23 & 8.72 & 9.84 \\
\hline
\end{tabular}

December at the TUG site with a median Fried parameter of $6.12 \mathrm{~cm}\left(q_{1}=4.18, q_{3}=8.70\right)$. Here, $q_{1}$ and $q_{3}$ are first and third quartiles, respectively. The lowest Fried parameters were measured in the period of July-September with a median Fried parameter of $3.56 \mathrm{~cm}\left(q_{1}=2.49, q_{3}=5.05\right)$. Median Fried parameter calculated from all observations made during our observation period is $4.48 \mathrm{~cm}\left(q_{1}=3.16, q_{3}=6.18\right)$.

The best day-time Fried parameters at the TUG site were obtained during the first three hours of the day as usual in solar observatories. The median Fried parameters for this time interval are $5.43\left(q_{1}=4.75, q_{3}=6.33\right), 4.82\left(q_{1}=4.23, q_{3}=5.75\right)$, $5.82\left(q_{1}=4.59, q_{3}=7.63\right), 5.98\left(q_{1}=4.56, q_{3}=8.21\right)$, $5.26\left(q_{1}=4.31, q_{3}=6.71\right), 4.59\left(q_{1}=3.51, q_{3}=6.59\right)$, $6.34\left(q_{1}=5.22, q_{3}=8.24\right), 5.07\left(q_{1}=4.08, q_{3}=6.25\right)$, $9.16\left(q_{1}=8.06, q_{3}=10.07\right) \mathrm{cm}$ for April, May, June, July, August, September, October, November and December, respectively.

Our observations demonstrate the dependence of median Fried parameter to the wind speed. The median Fried parameters are $4.51 \mathrm{~cm}\left(q_{1}=2.79, q_{3}=7.03\right)$ for $0-1 \mathrm{~m} / \mathrm{s}, 4.04 \mathrm{~cm}$ $\left(q_{1}=2.97, q_{3}=5.42\right)$ for $1-2 \mathrm{~m} / \mathrm{s}, 5.13 \mathrm{~cm}\left(q_{1}=3.99\right.$, $\left.q_{3}=6.63\right)$ for $2-3$ and $5.73 \mathrm{~cm}\left(q_{1}=5.09, q_{3}=7.08\right)$ for $>3 \mathrm{~m} / \mathrm{s}$ wind speeds. These values show that the best 
Table 4. Comparison of median Fried parameters $(\mathrm{cm})$ from ATST S-DIMM and TUG S-SIMM. EAM denotes Early AM, LAM Late AM, EPM Early PM and LPM Late PM. The TUG S-DIMM observations are very rare for the LPM part of the Apr-June period. ATST S-DIMM data was taken from ATST Site Survey Working Group with the helps of T. Rimmele and J. M. Beckers (2003).

\begin{tabular}{lccccc}
\hline \hline Period/Site & EAM & LAM & EPM & LPM & All Days \\
\hline Apr.-Jun. & & & & & \\
\hline Big Bear & 6.57 & 6.64 & 5.85 & 4.45 & 6.21 \\
Haleakala & 5.04 & 2.79 & 2.42 & 3.57 & 3.03 \\
La Palma & 5.49 & 3.48 & 3.17 & 3.42 & 3.65 \\
Panguitch Lake & 4.08 & 3.87 & 3.51 & 3.14 & 3.77 \\
Sacramento Peak & 4.86 & 2.65 & 1.93 & 2.13 & 2.40 \\
San Pedro Martir & 4.37 & 2.99 & 2.54 & 3.09 & 3.00 \\
TUG & 5.55 & 4.28 & 4.63 & - & 4.89 \\
\hline Jul. - Sep. & & & & & \\
\hline Big Bear & 6.34 & 7.13 & 6.50 & 4.93 & 6.45 \\
Haleakala & 5.40 & 3.12 & 2.71 & 3.53 & 3.33 \\
La Palma & 5.47 & 3.55 & 3.22 & 3.72 & 3.73 \\
Panguitch Lake & 4.15 & 3.63 & 2.92 & 3.53 & 3.59 \\
Sacramento Peak & 5.16 & 2.65 & 2.05 & 2.19 & 3.13 \\
San Pedro Martir & 3.91 & 2.66 & 2.19 & 2.42 & 2.73 \\
TUG & 5.36 & 3.16 & 2.65 & 2.83 & 3.56 \\
\hline Oct. - Dec. & & & & & \\
\hline Big Bear & 5.28 & 6.61 & 5.71 & 5.44 & 5.93 \\
Haleakala & 5.86 & 3.58 & 2.74 & 3.38 & 3.57 \\
La Palma & 4.08 & 2.90 & 2.66 & 3.20 & 2.96 \\
Panguitch Lake & 2.48 & 2.88 & 2.79 & 2.75 & 2.77 \\
Sacramento Peak & 4.90 & 2.96 & 2.20 & 2.58 & 2.72 \\
San Pedro Martir & - & - & - & - & - \\
TUG & 6.95 & 6.40 & 5.15 & 4.73 & 6.12 \\
\hline & & & & & \\
\hline
\end{tabular}

day-time seeing conditions occur for higher wind speeds at the TUG site. This is an interesting result showing the difference between day-time and night-time seeing conditions, because Ehgamberdiev et al. (2000) found that the best night-time seeing conditions occur for wind speeds lower than $5 \mathrm{~m} / \mathrm{s}$ at the Maidanak Observatory in Uzbekistan.

Site testing studies for the 4 meter US Advanced Technology Solar Telescope (ATST) allow a very valuable set of Fried parameters measured with S-DIMM in six observatory sites for about 18 months. It is interesting to note that the heights of the ATST S-DIMM and TUG S-DIMM from the ground are 8 and 7 meters, respectively, that is almost the same. Therefore, keeping in mind that the Fried parameter increases with the height, a comparison of the median Fried parameters obtained in the ATST candidate sites with those of TUG can give an idea about the seeing quality of the TUG site. This comparison is summarized in Table 4 for the period of April-June, July-September and October-December. In this table, EAM means the first time interval of $3 \mathrm{~h}$ after sunrise, and the other columns follow each other with $3 \mathrm{~h}$ durations. A comparison of the median Fried parameters reveals that the median Fried parameters obtained at the TUG site can be comparable with those of the observatory sites listed in Table 4.

\section{Conclusion}

We constructed a differential image motion monitor that, with its good instrumental parameters and portability, can be used both for night-time and day-time seeing observations at the TUG site. Since it is a portable instrument, TUG S-DIMM can be used for site testing studies at any site.

By considering the results summarized above, we can conclude that the seeing conditions at the TUG site are rather good compared to those of good solar observatory sites from the point of view of measured Fried parameters, at least for our observation period. Day-time seeing observations over the future years are needed to confirm this result.

The data presented in this study were collected over too short a period to demonstrate long-term variations, and even short-term variations were not well sampled. In addition, results of this study are obtained from a new measurement technique that is still being developed and tested for day-time seeing studies, although it has been used for night-time seeing studies for about 10 years in many observatory sites. Thus, we consider these results as a general representation of the daytime seeing conditions at the TUG site.

Acknowledgements. This project was supported by TÜBİTAK (Turkish Scientific and Technical Research Council), Project Number: TBAG-2217 (102T110). We wish to thank Dr. Alexander Yascovich for his efforts in obtaining an optical wedge and for his interest. We are greateful to J. M. Beckers and T. Rimmele for the ATST S-DIMM data. We would also like to thank the staff of the TÜBITTAK National Observatory and the staff of the Solar Physics Section at the İstanbul University Observatory for their comments and technical support. The authors are greateful to the referee M. Sarazin for his valuable comments.

\section{References}

Ambastha, A., Bhatnagar, A., Rajmal, J., et al. 1991, Bull. Astr. Soc. India, 19, 215

Bally, J., Theil, D., Billawala, Y., et al. 1996, PASA, 13, 22

Beckers, J. M. 1998, SPIE Proc., 3352, 588

Beckers, J. M. 2001, Exp. Astron., 12, 1

Ehgamberdiev, S. A., Baijumanov, A. K., Ilyasov, S. P., et al. 2000, A\&AS, 145, 293

Fried, D. 1965, J. Opt. Soc. Am., 55, 1427

Hill, F., Fischer, G., Forgach, S., et al. 1994, Sol. Phys., 152, 351

Keil, S. L., Rimmele, T., \& Keller, C. U. 2002, SPIE Proc., 4853, 36

Liu, Z., \& Beckers, J. M. 2000, Sol. Phys., 198, 197

Martin, H. M. 1987, PASP, 99, 1360

Rimmele, T., \& Beckers, J. M. 2003, private communication, ATST Site Survey Working Group, ATST Project

Sarazin, M., \& Roddier, F. 1990, A\&A, 227, 294

Stock, J., \& Keller, G. 1960, in Stars and Stellar Systems, Telescopes, ed. G. P. Kuiper, \& B. M. Middelhurst (Chicago: Univ. Chicago Press), 1,138

Tokovinin, A. 2002, PASP, 114, 1156

Vernin, J., \& Mun̄oz-Tūonon, C. 1995, PASP, 107, 265 